\title{
Barriers to recruitment into a multi-site randomized controlled trial comparing the effectiveness of specialty outpatient versus nurse-led telephonic palliative care of older adults with advanced illness
}

\author{
Julia A Brickey \\ University of North Carolina School of Medicine \\ Jeanne Cho ( $\nabla$ jeanne.cho@nyulangone.org ) \\ NYU Langone Health https://orcid.org/0000-0002-3357-7012 \\ Mara Flannery \\ NYU Langone Health \\ Corita R. Grudzen \\ NYU Langone Health \\ EMPallA Investigators \\ NYU Langone Health
}

\section{Research article}

Keywords: Patient Recruitment, Palliative Medicine, Emergency medicine, Recruitment Barriers

Posted Date: April 7th, 2020

DOI: https://doi.org/10.21203/rs.3.rs-21202/v1

License: (c) (1) This work is licensed under a Creative Commons Attribution 4.0 International License.

Read Full License 


\section{Abstract}

Background Emergency department (ED) visits among older adults are common near the end of life. Palliative care has been shown to reduce ED visits and to increase quality of life among patients, but recruitment into these programs is often challenging.

Methods A multi-site randomized controlled trial investigating two modes of community-based palliative care delivery for patients in the ED who are discharged home. Research coordinators monitored factors that prevented eligibility and enrollment by screening electronic health records to identify and recruit eligible patients and their caregivers. Reasons for ineligibility and non-participation were documented.

Results Among the 7,091 patients who met the age and disease qualifier, 4,292 (60.5\%) were deemed ineligible due to hospital admission. Additional reasons for ineligibility included previous palliative care (919 [13\%]) or residence in a nursing facility $(462$ [6.5\%]). Of the 695 patients who were eligible, 298 were enrolled, yielding an enrollment rate of $43 \%$ (range of $9 \%$ to $80 \%$ across all sites). Of the 397 eligible patients who did not participate, $180(45.3 \%)$ refused due to barriers related to illness severity. Patients commonly refused due to misconceptions/stigma related to palliative care (118 [29.7\%]). One-hundred fifteen patients $(29 \%)$ refused due to the mode of palliative care delivery.

Conclusions Hospital admission from the ED proved to be the most significant barrier to eligibility. Eligible patients often refused participation due to the nature of their illness, misconceptions about palliative care, and concerns about being able to attend clinic visits if assigned to the outpatient arm.

\section{Background}

Multiple studies have shown that palliative care, which may be initiated in the emergency department (ED) and delivered in conjunction with life-prolonging treatment, improves quality of life in patients with advanced illness. ${ }^{1-3}$ Additionally, patients receiving palliative care utilize fewer resources, have reduced ED visits, and fewer admissions, leading to reduced cost of medical care and higher satisfaction for patients and families. ${ }^{2,4}$ In spite of this, there are significant barriers to enrolling patients in palliative care programs. These barriers include patient, family, and provider refusal, difficulties in communication regarding end-of-life care, and lack of training in palliative care amongst providers. ${ }^{5-8}$ Recruiting patients into palliative care research in the ED creates additional challenges due to the fast-paced environment, lack of an ongoing relationship with the patient, and high symptom burden that brought patients there to begin with. ${ }^{9,10}$

Engaging older adults with advanced illness in research is challenging for multiple reasons. Patients are often too sick or burdened by their illness to participate, and limited life-expectancy among this population complicates data collection. ${ }^{11,12}$ In spite of these issues, the research that does exist highlights the overwhelmingly positive benefits of palliative care in patients with serious illnesses. ${ }^{1,2,13}$ 
This knowledge, combined with the lack of understanding about how best to deliver palliative care to patients, warrants the need for additional research.

This study is a five-year, randomized controlled trial designed to compare the effectiveness of two modes of community-based palliative care delivery for patients with advanced illness who are recruited from the ED or observation unit: nurse-led telephonic supportive care and facilitated, outpatient specialty palliative care. One year into the study, there have been significant barriers to both eligibility and recruitment at nine different ED sites across the United States. The focus of this paper is to assess the barriers to enrolling patients with advanced illness into a palliative care randomized controlled trial in the ED setting.

\section{Methods}

Research coordinators monitored factors that prevented patient enrollment into Emergency Medicine Palliative Care Access (EMPallA), a multi-site randomized controlled trial (RCT) comparing two methods of delivering community-based palliative care. ${ }^{14}$ EMPallA currently recruits patients and their caregivers from nine ED sites across the country. Eligibility criteria for patients include those who are 50 years of age or older who present to the ED with a qualifying illness. Qualifying illnesses include advanced cancer or end-stage organ failure (defined as New York Heart Association Class III or IV heart failure, End-Stage Renal Disease with glomerular filtration rate $<15 \mathrm{ml} / \mathrm{min} / \mathrm{m}^{2}$, or Global Initiative for Chronic Obstructive Lung Disease Stage III, IV, or oxygen-dependent Chronic Obstructive Pulmonary Disease) among patients who are scheduled for ED discharge or observation status. Patients must also have health insurance, speak English or Spanish, and reside within the geographic area. Patients are excluded from the study if they have previously received hospice or palliative care, are admitted to inpatient services, have dementia, or live in a skilled nursing facility (SNF) or similar assisted living facility (ALF). Caregivers who are at least 18 years old, have access to a working telephone, and speak English or Spanish are also eligible to enroll in the study. The study was submitted to and approved by the NYU School of Medicine Institutional Review Board Ethics Committee.

Research coordinators screened electronic health records from 7am-12am, six days a week to identify potentially eligible patients. If a patient met study criteria, research coordinators approached the patient and obtained informed consent at bedside. If a caregiver was present at the time, they were also invited to enroll in the study. Every patient or caregiver who enrolled and completed the baseline survey received at $\$ 40$ gift card as study compensation. All study procedures have been approved by the Institutional Review Board at each institution.

During the daily electronic health record screens, research coordinators documented reasons for noneligibility and refusals. For patients who met one or more of the disease qualifiers but were determined ineligible, the reasons were documented. Patients could be ineligible for more than one reason (e.g. have dementia and live in a SNF). For patients who were eligible but chose not to participate in the study, they were asked to provide a reason for their refusal. Research coordinators then recorded their response using a pre-determined list of common reasons for refusal. Patients were permitted to provide more than one 
reason. Research coordinators recorded all data in Research Electronic Data Capture (REDCap), a secure, web-based application that served as the research database. In order to track eligibility and enrollment, refusal data was organized into a Consolidated Standards for Reporting of Trials (CONSORT) diagram (see Appendix A). ${ }^{15-17}$

\section{Results}

To date, 7,091 patients with a qualifying illness have been identified, of which 6,196 were deemed ineligible for the study (87.3\%). Table 1 provides a comprehensive list of reasons for patients who met the disease qualifier were deemed ineligible for the study. Across all sites, 4,292 (60.5\%) patients were ineligible due to hospital admission from the ED (range: $38-82.1 \%$ across all sites). Other common reasons included previous exposure to palliative care (13\%) and residence in a SNF or ALF (6.5\%). A smaller proportion of patients spoke a primary language other than English or Spanish (4.9\%) or were diagnosed with dementia (4.1\%). Other barriers included living outside the treatment area, lack of insurance, and current hospice care, but these were less common $(<2 \%)$.

Table 1

Reasons for non-eligibility among patients who met disease qualifier $(n=7,091)$.

\begin{tabular}{|ll|}
\hline Reason for non-eligibility & Patients, no. (\%)* \\
\hline Admitted to inpatient services & $4292(60.5)$ \\
\hline Previous palliative care & $919(13.0)$ \\
\hline Lives in SNF/ALF & $462(6.5)$ \\
\hline Primary language not English or Spanish & $346(4.9)$ \\
\hline Dementia & $293(4.1)$ \\
\hline Does not live within treatment area & $123(1.7)$ \\
\hline Previous hospice care & $125(1.8)$ \\
\hline Unaware of cancer diagnosis & $66(0.9)$ \\
\hline Uninsured & $44(0.6)$ \\
\hline *Does not add up to 100\% because patients may have been eligible for more than one reason. \\
\hline
\end{tabular}

Of the 695 eligible patients, 298 (43\%) were successfully enrolled. Enrollment rates varied by site ranging from $9-80 \%$. Sample sizes were widely variable (see Table 2). Caregiver enrollment was also tracked by site. Of the 298 patients enrolled, 85 also had a caregiver enrolled, resulting in a caregiver enrollment rate of $29 \%$ (range: $0-75 \%$ across all sites). 
Table 2

Patient enrollment rate by ED site.

\begin{tabular}{|lllll|}
\hline $\begin{array}{l}\text { ED } \\
\text { site }\end{array}$ & $\begin{array}{l}\text { Total eligible } \\
\text { patients }\end{array}$ & $\begin{array}{l}\text { Total patients } \\
\text { refused }\end{array}$ & $\begin{array}{l}\text { Total patients } \\
\text { enrolled }\end{array}$ & $\begin{array}{l}\text { Patient enrollment } \\
\text { rate }\end{array}$ \\
\hline A & 79 & 16 & 63 & $80 \%$ \\
\hline B & 19 & 6 & 13 & $68 \%$ \\
\hline C & 11 & 4 & 7 & $64 \%$ \\
\hline D & 83 & 38 & 45 & $54 \%$ \\
\hline E & 71 & 38 & 33 & $46 \%$ \\
\hline F & 113 & 65 & 48 & $42 \%$ \\
\hline G & 210 & 131 & 79 & $38 \%$ \\
\hline H & 109 & 99 & 10 & $9 \%$ \\
\hline Total & 695 & 397 & 298 & $43 \%$ \\
\hline
\end{tabular}

Reasons for refusal were categorized as follows: (1) barriers related to illness severity, (2) misconceptions/stigma related to palliative care, (3) mode of palliative care delivery, (4) general research barriers, and (5) family/caregiver barriers. Results are based on the number of patients that cited each reason, and patients were allowed to give more than one reason. Table 3 outlines the frequency of each refusal by category.

One-hundred fifteen patients (29\%) refused due to the mode of palliative care delivery. The overwhelming majority of these patients (99 [86.1\%]) did not want to or could not commit to attending the outpatient clinic visits. In contrast, only 16 patients (13.9\%) stated that they did not want to receive telephonic palliative care. In some instances, patients wanted the choice to be placed in one intervention arm over another but refused study participation since they could not be guaranteed placement into their preferred treatment arm.

Sixty patients (15.1\%) refused to participate for reasons categorized as "general research barriers." Patients typically exhibited a lack of interest in research participation (54 [90.0\%]). However, some patients reported a fear of privacy breach or had negative feelings towards research (6 [10.0\%]). A smaller proportion of patients did not participate due to family or caregiver barriers (40 [10.1\%]). In some cases, a family member or caregiver recommended the patient not participate $(24[60.0 \%])$. Sixteen patients $(40 \%)$ reported that they did not want to make a decision without a family member or a caregiver present.

\section{Discussion}

\section{Main Findings}


Multiple barriers prevented both patient eligibility and recruitment into community-based palliative care within the context of a multi-site, five-year RCT. Hospital admission from the ED was the main reason for ineligibility. This exclusion criterion is essential in order for EMPallA to target a specific group of patients who may not otherwise have access to palliative care programs. Notably, palliative care services are available to admitted patients, but few resources for patients who are discharged home from the ED. ${ }^{18-20}$ Although admission from the ED prevented many patients from being eligible for the study, this criterion is essential in order to address this gap in palliative care services.

Enrollment rate varied widely by site, from $9-80 \%$ of eligible patients agreeing to participate. This variation is likely the result of multiple factors, such as varying experience and comfort level of the research coordinator discussing palliative care, differing levels of stigma around palliative care, and the variable proximity of outpatient clinics. The research coordinator's experience recruiting participants and comfort level in having conversations related to end-of-life care may vary given the difficult nature of this topic. These conversations can be challenging for healthcare personnel as well, especially in a busy ED setting and all the more intimidating for research coordinators. ${ }^{6}$ In addition, it is possible that stigma related to palliative care may vary based on geographic location. A study utilizing the Dartmouth Atlas of Healthcare highlighted regional variation in end-of-life care-with greater healthcare utilization at the end of life in the South and along the urban corridors on the East and West Coasts when compared with Midwestern and Mountain regions. ${ }^{21}$ These demonstrated differences in healthcare utilization at the end of life may be associated with differences in patient and family receptiveness to palliative care. Finally, proximity to healthcare and outpatient facilities and accessibility to transportation differed among the sites, which may have influenced a patient's willingness to participate.

As shown in previous studies of populations with advanced illness, patients commonly declined participation for reasons that were related to living with an advanced illness, such as feeling overburdened with their current care. The FamCope intervention, which aimed to test the feasibility of nurse-led, family-coping-oriented palliative home care in patients with advanced cancer, also reported a high refusal rate related to the characteristics of their target population. ${ }^{5}$ Many of these patients refused because their illness burden was too great, often citing a "lack of energy" or being "too sick." It is well documented that research with palliative care populations is inherently difficult due to the aforementioned reasons, so these findings did not come as a surprise. ${ }^{11,22,23}$ Among the eligible patients in our study population, some felt that they were satisfied with their current care. This could be explained by the chronic nature of their illness, which may require that these patients see several specialists, attend frequent appointments, and receive in-home care.

Recruitment barriers related to misconceptions or stigma regarding palliative care were also common. Patients often associated palliative care with hospice or death. Although it is well documented in the literature that palliative care improves quality of life and symptom burden for patients and their caregivers, patients often had preconceived notions about palliative care that could not be alleviated even after research coordinators described palliative care and its potential benefits. 


\section{Future Implications}

Mode of palliative care delivery was an influential factor for patients' decisions to participate in the study. Of the 115 patients who did not participate for this reason, $99(86.1 \%)$ of them indicated that they did not want outpatient palliative due to inability or unwillingness to make it to the clinic visits. In contrast, only $16(13.9 \%)$ said that they did not want to receive telephonic palliative care. These findings suggest that patients may be more open to receiving palliative care if they did not have to attend outpatient clinic visits, which will have important implications for scalability.

In contrast to previous studies which have shown that caregivers often act as gatekeepers and refuse on behalf of the patient, barriers due to family or caregiver refusal were among the least common for our study. ${ }^{23,24}$ This may be explained by the fact that many of our patients were alone at the time they were approached. Research coordinators typically approached patients during the weekdays, so caregivers who have jobs may be less likely to be present. Geographic variation likely also plays a role in these findings. Many of our sites are located in urban areas with public transportation or public assistance programs, making it easier for patients to access healthcare without a caregiver.

Furthermore, studies have documented that physician gatekeeping is a common barrier to patient recruitment, particularly with palliative care populations. ${ }^{10,22}$ On the contrary, this study has not yet encountered physician refusal. This may be a direct result of the requirement that physicians opt out at the beginning of the study, as per study protocol. It may also reflect changing attitudes toward palliative care. A study analyzing the perceptions of palliative care among healthcare providers before and after implementation of a palliative medicine division found increased attendance in educational activities and increased confidence in palliative care. ${ }^{25}$ Researchers also noted that providers who favored comanagement with palliative care held core values that aligned with current concepts, such as advanced

care planning ${ }^{25}$ As the number of palliative care programs in hospitals across the country increases, we expect to see a similar increase in understanding and acceptance of palliative care amongst providers. ${ }^{26}$

\section{Strengths/limitations}

The strength of this paper is the generalizability of the results across different geographic ED contexts and patient populations with advanced illness globally. Given that this is a descriptive paper that is exploratory in nature, one of the limitations is that the results cannot be used to draw definitive conclusions. We report on data that could be hypothesis-generating for researchers and clinicians in the field. Additionally, the small sample sizes at some of our ED sites limit our ability to comment on these results. For example, one of the study sites has a fairly high enrollment rate at $68 \%(n=13$ of 19$)$. However, with less than 20 eligible patients to date, it is likely that this number will change as the study progresses.

\section{Conclusion}


Hospital admission from the ED is common among our population, which proved to be our most significant barrier to eligibility. Enrollment rates vary widely by site, likely due to site-specific barriers. Patients who were eligible often refused participation due to the nature of their illness, misconceptions about palliative care, and concerns about being able to attend clinic visits if assigned to the outpatient arm.

\section{List Of Abbreviations}

Assisted Living Facility (ALF); Consolidated Standards for Reporting of Trials (CONSORT); Emergency Department (ED); Emergency Medicine Palliative Care Access (EMPallA); Randomized Controlled Trial (RCT); Research Electronic Data Capture (REDCap); Skilled Nursing Facility (SNF)

\section{Declarations}

Ethics approval and consent to participate: Approved by the New York University School of Medicine Institutional Review Board.

Consent to participate: written informed consent was obtained from study participants.

Availability of data and materials: Data sharing is not applicable to this article as no datasets were generated or analyzed during the current study.

Competing interests: The authors declare that they have no competing interests.

Funding: This work was supported through a Patient-Centered Outcomes Research Institute (PCORI) Award (PLC-1609-36306). PCORI has been involved in the design of the study and collection.

Authors' contributions: JB: drafting and critically revising the manuscript. JC: drafting and critically revising the manuscript. MF: drafting and critically revising the manuscript. CRG: drafting and critically revising the manuscript. The EMPallA Investigators' development of the protocol and manuscript review. All authors approved of the final version of the manuscript.

Acknowledgements: We would like to thank the members of the EMPallA Study Advisory Committee and Allison Cuthel for their contributions to the study recruitment protocol and processes.

\section{References}

1. Brumley RD, Enguidanos S, Cherin DA. Effectiveness of a Home-Based Palliative Care Program for End-of-Life. J Palliat Med. 2003;6(5):715-724. doi:10.1089/109662103322515220

2. Brumley R, Enguidanos $S$, Jamison $P$, et al. Increased satisfaction with care and lower costs: Results of a randomized trial of in-home palliative care. J Am Geriatr Soc. 2007;55(7):993-1000.

doi:10.1111/j.1532-5415.2007.01234.x 
3. Strömgren AS, Sjogren P, Goldschmidt D, et al. A longitudinal study of palliative care: Patientevaluated outcome and impact of attrition. Cancer. 2005;103(8):1747-1755. doi:10.1002/cncr.20958

4. Riegel B, Carlson B, Kopp Z, LePetri B, Glaser D, Unger A. Effect of a standardized nurse casemanagement telephone intervention on resource use in patients with chronic heart failure. Arch Intern Med. 2002;162(6):705-712. http://www.ncbi.nlm.nih.gov/pubmed/11911726.

5. Ammari ABH, Hendriksen C, Rydahl-Hansen S. Recruitment and Reasons for Non-Participation in a Family-Coping-Orientated Palliative Home Care Trial (FamCope). J Psychosoc Oncol. 2015;33(6):655-674. doi:10.1080/07347332.2015.1082168

6. Smith AK, Fisher J, Schonberg MA, et al. Am I Doing the Right Thing? Provider Perspectives on Improving Palliative Care in the Emergency Department. Ann Emerg Med. 2009;54(1):86-93.e1. doi:10.1016/j.annemergmed.2008.08.022

7. Anselm AH, Palda V, Guest CB, et al. Barriers to communication regarding end-of-life care: Perspectives of care providers. J Crit Care. 2005;20(3):214-223. doi:10.1016/j.jcrc.2005.05.012

8. Kelley AS, Morrison RS. Palliative Care for the Seriously III. N Engl J Med. 2015;373(8):747-755. doi:10.1056/nejmra1404684

9. Marcelin I, McNaughton C, Tang N, Caterino J, Grudzen C. Palliative Care Needs of Advanced Cancer Patients in the Emergency Department (S767). J Pain Symptom Manage. 2018;55(2):693. doi:10.1016/j.jpainsymman.2017.12.421

10. Grudzen CR, Richardson LD, Kandarian B, Ortiz J, Copeli N, Morrison RS. Barriers to palliative care research for emergency department patients with advanced cancer. J community Support Oncol. 2014;12(5):158-162. doi:10.12788/jcso.0040.158

11. Rinck GC, van den Bos GAM, Kleijnen J, de Haes HJCJM, Schadé E, Veenhof CHN. Methodologic issues in effectiveness research on palliative cancer care: A systematic review. J Clin Oncol. 1997;15(4):1697-1707. doi:10.1200/JC0.1997.15.4.1697

12. Fischer SM, Gozansky WS, Sauaia A, Min SJ, Kutner JS, Kramer A. A Practical Tool to Identify Patients Who May Benefit from a Palliative Approach: The CARING Criteria. J Pain Symptom Manage. 2006;31(4):285-292. doi:10.1016/j.jpainsymman.2005.08.012

13. Smith TJ, Coyne P, Cassel B, Penberthy L, Hopson A, Hager MA. A High-Volume Specialist Palliative Care Unit and Team May Reduce In-Hospital End-of-Life Care Costs. J Palliat Med. 2003;6(5):699705. doi:10.1089/109662103322515202

14. Grudzen CR, Shim DJ, Schmucker AM, Cho J, Goldfeld KS. Emergency Medicine Palliative Care Access (EMPallA): Protocol for a multicentre randomised controlled trial comparing the effectiveness of specialty outpatient versus nurse-led telephonic palliative care of older adults with advanced illness. BMJ Open. 2019;9(1):1-8. doi:10.1136/bmjopen-2018-025692

15. Kane RL, Wang J, Garrard J. Reporting in randomized clinical trials improved after adoption of the CONSORT statement. J Clin Epidemiol. 2007;60(3):241-249. doi:10.1016/j.jclinepi.2006.06.016

16. Begg C, Cho M, Eastwood S, et al. of Randomized Controlled Trials The CONSORT Statement. Jama. 1996;276(8):637-639. doi:10.1001/jama.1996.03540080059030 
17. Toerien $\mathrm{M}$, Brookes ST, Metcalfe $\mathrm{C}$, et al. A review of reporting of participant recruitment and retention in RCTs in six major journals. Trials. 2009;10:1-12. doi:10.1186/1745-6215-10-52

18. Kripalani S, Theobald CN, Anctil B, Vasilevskis EE. Reducing Hospital Readmission Rates: Current Strategies and Future Directions. Annu Rev Med. 2013;65(1):471-485. doi:10.1146/annurev-med022613-090415

19. Williams M V. A requirement to reduce readmissions: Take care of the patient, not just the disease. JAMA - J Am Med Assoc. 2013;309(4):394-396. doi:10.1001/jama.2012.233964

20. Hansen L, Young R, Hinami K, Leung A, Williams M. Interventions to Reduce 30-Day Rehospitilization: A Systematic Review. Ann Intern Med. 2013;155(Improving Patient Care):520-528. doi:doi: 10.7326/0003-4819-155-8-201110180-00008.

21. Gessert CE, Haller I V, Johnson BP. Regional variation in care at the end of life: discontinuation of dialysis. 2013:11-20.

22. Ewing G, Rogers M, Barclay S, McCabe J, Martin A, Todd C. Recruiting patients into a primary care based study of palliative care: Why is it so difficult? Palliat Med. 2004;18(5):452-459. doi:10.1191/0269216304pm905oa

23. Wohleber AM, McKitrick DS, Davis SE. Designing Research With Hospice and Palliative Care Populations. Am J Hosp Palliat Med. 2012;29(5):335-345. doi:10.1177/1049909111427139

24. Williams CJ, Shuster JL, Clay OJ, Burgio KL. Interest in Research Participation among Hospice Patients, Caregivers, and Ambulatory Senior Citizens: Practical Barriers or Ethical Contraints? J Palliat Med. 2006;9(4):968-974. doi:10.1089/jpm.2006.9.968

25. Cheung NY, Gorelik A, Mehta P, et al. Perception of palliative medicine by health care professionals at a teaching community hospital: what is the key to a "palliative attitude"? J Multidiscip Healthc. 2019; Volume 12:437-443. doi:10.2147/jmdh.s182356

26. Dumanovsky T, Augustin R, Rogers M, Lettang K, Meier DE, Morrison RS. The Growth of Palliative Care in U.S. Hospitals: A Status Report. J Palliat Med. 2016;19(1):8-15. doi:10.1089/jpm.2015.0351

\section{Supplementary Files}

This is a list of supplementary files associated with this preprint. Click to download.

- RecruitmentBarriersAppendixCONSORTBMCPall3420.pdf

- EMPallAResearchInvestigatorList2020.xIsx 
Table 3

Reasons that eligible patients did not participate $(n=397)$.

\begin{tabular}{|ll|}
\hline Reason for non-participation & Patients, no. (\%) \\
\hline Barriers related to illness severity & $180(45.3)$ \\
\hline $\begin{array}{l}\neg \text { Too burdened by other appointments/not enough time } \\
\neg \text { Satisfied with current care } \\
\neg \text { Doesn't feel well enough to participate }\end{array}$ & $82(45.6)$ \\
\hline Misconceptions/stigma related to palliative care & $30(17.8)$ \\
\hline$\neg$ Doesn't feel illness is serious enough & $118(29.7)$ \\
$\neg$ Associates palliative care with hospice and/or death & $63(53.4)$ \\
\hline Mode of palliative care delivery & $55(46.6)$ \\
\hline$\neg$ Doesn't want outpatient palliative care & $115(29.0)$ \\
$\neg$ Doesn't want telephonic palliative care & $99(86.1)$ \\
\hline General research barriers & $16(13.9)$ \\
\hline$\neg$ Lack of interest in research participation & $60(15.1)$ \\
$\neg$ Fear of privacy breach/negative feelings towards research & $54(90.0)$ \\
\hline Family/caregiver barriers & $6(10.0)$ \\
\hline$\neg$ Family declined patient's participation & $40(10.1)$ \\
\hline$\neg$ Doesn't want to make decision without family/caregiver present & $24(60.0)$ \\
\hline$*$ Does not add up to 100\% because patients may have declined participation for multiple reasons. \\
\hline
\end{tabular}

